\title{
The Meaning of Interaction
}

\author{
Xuefeng Wang Robert C. Elston Xiaofeng Zhu \\ Department of Epidemiology and Biostatistics, Case Western Reserve University, Cleveland, Ohio, USA
}

\section{Key Words}

Epistasis · Gametic phase disequilibrium • Interaction •

Transformation

\begin{abstract}
Although recent studies have attempted to dispel the confusion that exists in regard to the definition, analysis and interpretation of interaction in genetics, there still remain aspects that are poorly understood by non-statisticians. After a brief discussion of the definition of gene-gene interaction, the main part of this study addresses the fundamental meaning of statistical interaction and its relationship to measurement scale, disproportionate sample sizes in the cells of a two-way table and gametic phase disequilibrium.
\end{abstract}

Copyright $\odot 2010$ S. Karger AG, Base

\section{Introduction}

There has been a long-standing interest in the investigation of interactions in genetics, including gene-environment and gene-gene interactions, based on the assumption that they play an important role in the etiology of complex diseases or traits; however, often there is no clear definition of what is being sought. The advent of large-scale human association studies has further stimulated the development of new methods of statistical analysis in the hope of discovering gene-gene interactions or prioritizing single-nucleotide polymorphisms (SNPs) [1]. Recently, a flurry of work has been devoted to related computational issues surrounding high dimensionality and multiple testing, resulting in the emergence of new methods such as those based on data reduction and data mining techniques $[2,3]$. Yet, the real challenge - also a major reason why many people remain skeptical of the usefulness of statistical findings - remains how to justify and interpret the statistical interaction models and integrate the results with biological mechanisms. In fact, the generic nature of the term 'interaction' has introduced considerable confusion into every step from definition to analysis and interpretation. Our main purpose here, therefore, is to discuss in some detail the fundamental meaning of interaction, carefully differentiating the biological and statistical aspects, especially in so far as the term relates to human studies.

According to Webster's dictionary, interaction has two meanings: intermediate action and 'action on each other; reciprocal action or effect'. This latter meaning suggests social interaction and also what is thought of as biological interaction. There is, however, no universally accepted definition of interaction in either biology or statistics. In the broadest sense, the term only implies that objects or factors in a study do not act independently. Many 'working' definitions are directly based on the statistical characteristics or measures of interaction (again, no one measure being generally agreed upon), often causing misunderstanding, if not even more confusion. To reduce

\section{KARGER}

(C) 2010 S. Karger AG, Basel

Fax +41613061234 E-Mail karger@karger.ch www.karger.com www.karger.com/hhe

\section{Dr. R.C. Elston}

Case Western Reserve University

2103 Cornell Road, 1304

Cleveland, OH 44106-7281 (USA)

Tel. +1 216368 5630, Fax +1 216368 880, E-Mail robert.elston@cwru.edu 
Table 1. Alternative meanings given to some 'interaction' terms

\begin{tabular}{|c|c|c|}
\hline & I & II \\
\hline $\begin{array}{l}\text { Additive } \\
\text { interaction }\end{array}$ & $\begin{array}{l}\text { Statistical interaction measured on an additive scale, } \\
\text { i.e. the combined effect (risk) of the factors is higher or } \\
\text { less than the addition of the individual main effects. }\end{array}$ & $\begin{array}{l}\text { In some contexts, like evaluating the efficacy of two } \\
\text { medicines, the combined effect is equal to the sum of } \\
\text { the effects of the medicines given separately. }\end{array}$ \\
\hline $\begin{array}{l}\text { Epistatic } \\
\text { interaction }\end{array}$ & $\begin{array}{l}\text { One gene (or any genetic factor) masks or suppresses } \\
\text { the effect/action of other(s). }\end{array}$ & $\begin{array}{l}\text { Like 'epistasis', broadly implies any type of statistical/ } \\
\text { physical interactions between genetic factors. }\end{array}$ \\
\hline $\begin{array}{l}\text { Intragenic } \\
\text { interaction }\end{array}$ & Interaction between different SNPs within a gene. & $\begin{array}{l}\text { Interaction between alleles at the same locus } \\
\text { (dominance). }\end{array}$ \\
\hline $\begin{array}{l}\text { Multiplicative } \\
\text { interaction }\end{array}$ & Statistical interaction assessed on a multiplicative scale. & The product of main effects. \\
\hline $\begin{array}{l}\text { Physiological } \\
\text { epistasis/interaction }\end{array}$ & $\begin{array}{l}\text { Cheverud and Routman called interaction for } \\
\text { unweighted means 'physiological epistasis'. }\end{array}$ & $\begin{array}{l}\text { A biological process/mechanism similar to physical } \\
\text { interaction. }\end{array}$ \\
\hline $\begin{array}{l}\text { Quantitative } \\
\text { interaction }\end{array}$ & $\begin{array}{l}\text { The magnitude of the effects of one factor varies } \\
\text { across the levels of another, but not the direction } \\
\text { (removable/non-crossover interaction), contrasted } \\
\text { with 'qualitative interaction'. }\end{array}$ & Synonymous with 'statistical interaction.' \\
\hline $\begin{array}{l}\text { Synergistic/ } \\
\text { antagonistic } \\
\text { interaction }\end{array}$ & $\begin{array}{l}\text { The combined effect of the factors is greater/less than } \\
\text { the sum of the individual effects. }\end{array}$ & $\begin{array}{l}\text { The combined effect of the factors is greater/less than } \\
\text { any of the individual effects. }\end{array}$ \\
\hline $\begin{array}{l}\text { Statistical } \\
\text { epistasis/interaction }\end{array}$ & $\begin{array}{l}\text { Contrasted with 'physical interaction', } \\
\text { merely emphasizes its mathematical nature. }\end{array}$ & $\begin{array}{l}\text { Contrasted with 'compositional epistasis' [27] with a } \\
\text { special emphasis on its population-average property. }\end{array}$ \\
\hline
\end{tabular}

ambiguity, it is generally preferable to couple the term 'interaction' with other descriptive words or phrases, although many of these still have no clear-cut and generally accepted definitions (see table 1 for examples). The increased complexity of studies in human genetics makes them of necessity collaborative in nature, and collaborating researchers should always be crystal clear about the exact meaning of the words they use, because the same term (such as 'gene-gene interaction' and the terms listed in table 1) can convey quite different meanings to people with different scientific backgrounds.

\section{What Is Gene-Gene Interaction?}

Is gene-gene interaction the interaction between genes? From the standpoint of population genetics or genetic epidemiology, a frequently used definition of gene-gene interaction is the interaction between alleles at different loci. The term 'locus' is now being used not only for the location of a gene, but also (unfortunately, perhaps) for the location of any genetic variant or marker that is nearby or within a gene, which can serve as a surrogate for studying the actual gene. In genome-wide association studies (GWAS), routine analyses are mostly based on SNPs where a gene locus may involve hundreds or thousands of SNPs. The number of possible SNP pairs (let alone SNP trios, quartets, etc.) grows very rapidly with the number of genes - or SNPs in each gene - selected, posing a computational challenge and even greater difficulty in interpretation. Novel methods for tests at the gene or SNP set level are becoming available but are only beginning to be used in GWAS. To answer the question from the physical or molecular standpoint, it is essential to ask a more basic question that we often neglect: what is a gene? The central dogma states that genetic information flows from DNA to RNA to protein. But whereas $95 \%$ of all DNA is transcribed to RNA, very little of this is translated to protein. Physical interaction can be triggered at any stage. As conceptually illustrated in figure 1 , all the entities coexist and coordinate to form a dynamic cellular system. Two DNA variations may interact with each other directly, though, as far as is known, not very commonly. If we define a gene as any stretch of DNA with function [4], it is mostly gene products, not the genes themselves, that interact physically. DNA is packaged into chromatin fibers, which oc- 
Fig. 1. Conceptual model of physical genegene interaction. Most methods of detecting gene-gene interaction still rely on examining the relationship between phenotype and marker genotypes, where all the intermediate processes (the region enclosed by a dashed line) are treated as a black-box. However, physical interaction can occur at any stage, between any functional sequences/molecules. Although DNA-DNA interactions do exist, it is mostly the interactions between gene products that alter the final phenotypes, such as protein-protein, protein-DNA and protein-RNA interactions, each providing a new point of entry into understanding the complex physico-chemical gene-gene interaction system.

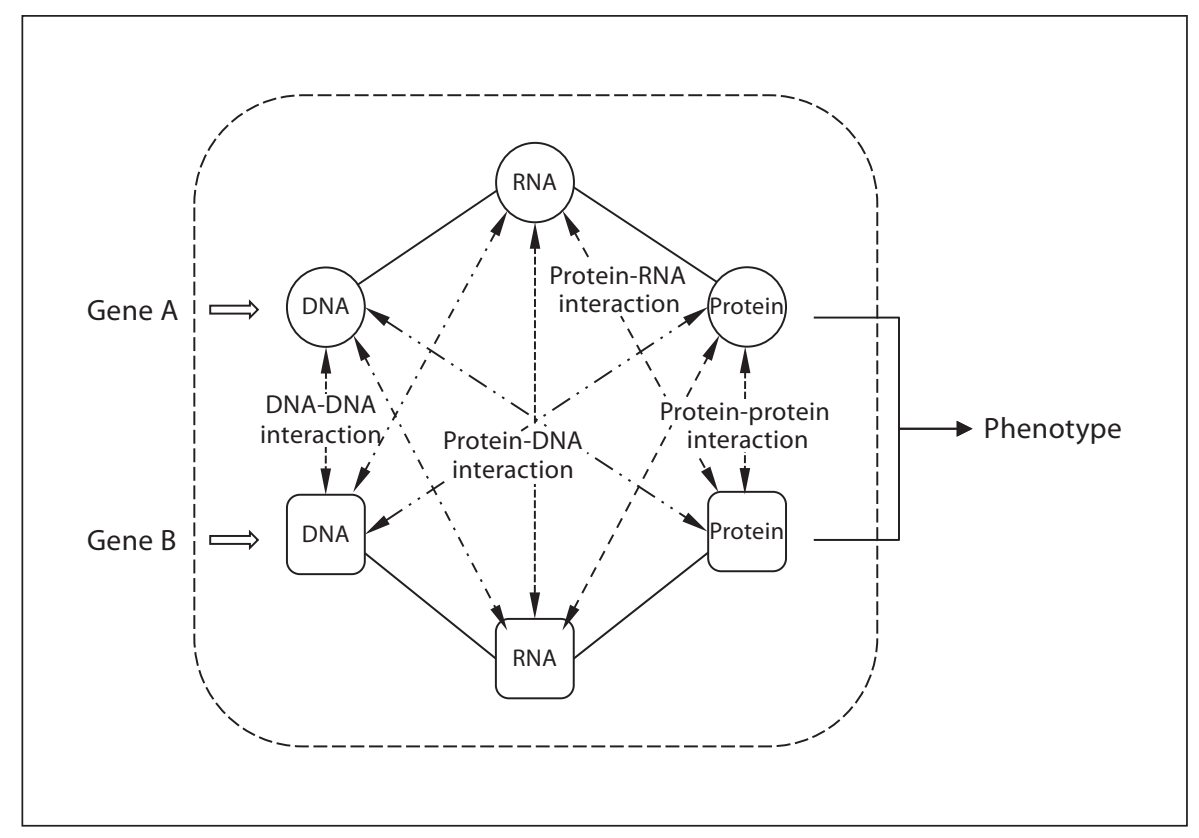

cupy distinct areas in the nucleus called 'chromosome territories' (CTs). Some regions of high gene density may loop out of CTs and move to contact other sites far from the region, causing an interesting event termed 'gene kissing' [5]. DNA can be specifically recognized by proteins with special structures (DNA binding motifs) to form protein-DNA complexes, which regulate both DNA replication and gene expression. Current knowledge about protein-RNA interactions, which are interactions between gene products, remains limited, but they are generally believed to be involved in RNA metabolism and translation. Considerable, perhaps disproportionate, attention has been given to the study of the interactions between proteins, including enzymes and other functional molecules that control various metabolic and regulatory pathways. This is largely driven by the availability of highthroughput proteomic techniques. Physical gene-gene interaction occurs relatively rarely as a fraction of all the physical events that jointly involve genes and their products, and these events occur irrespective of whether they have any effect on the final phenotypes. In the light of the modern definition of a gene [4] where final function is emphasized, it is irrational to give priority to protein-protein interactions rather than RNA-protein interactions. For what follows we explicitly (rather than implicitly, as is so often the case) define the term 'gene-gene interaction' to include gene-gene product and gene product-gene product interactions.
There is still much confusion between 'statistical genegene interaction' and 'biological gene-gene interaction', partly because of the use of the word 'epistasis' (epi = upon, stasis = stand). This word was coined over a century ago by Bateson [6], the same person who suggested the word 'genetics'. He used the word to describe only the masking action whereby an allele at a locus suppresses the effect of an allele at another locus, which results in a departure from the expected dihybrid ratio 9:3:3:1. The term later acquired a much broader meaning that is almost synonymous with gene-gene interaction as we have defined it. Fisher [7], in his seminal paper of 1918, used another noun form ('epistacy') to describe the deviations from the additive effects of alleles at different loci. This term was soon replaced by 'epistasis' in the quantitative genetics literature [8]. The meaning of interaction in genetics has now evolved into two rather divergent directions. 'Interaction' (or 'statistical interaction') is used by statisticians to describe the non-additivity in generalized linear models. This definition is thus close to Fisher's term 'epistacy'. Biologists use the term 'biological interaction', or simply 'interaction' to mean the joint action of two or more factors, whether or not an additive statistical model is sufficient, thinking of the physical interaction between molecules. We suggest that: (1) unless the mechanism is known, the term 'biological interaction' is better replaced by the less specific term 'joint action'; (2) 'interaction', if quantified, should be based on statistical con- 


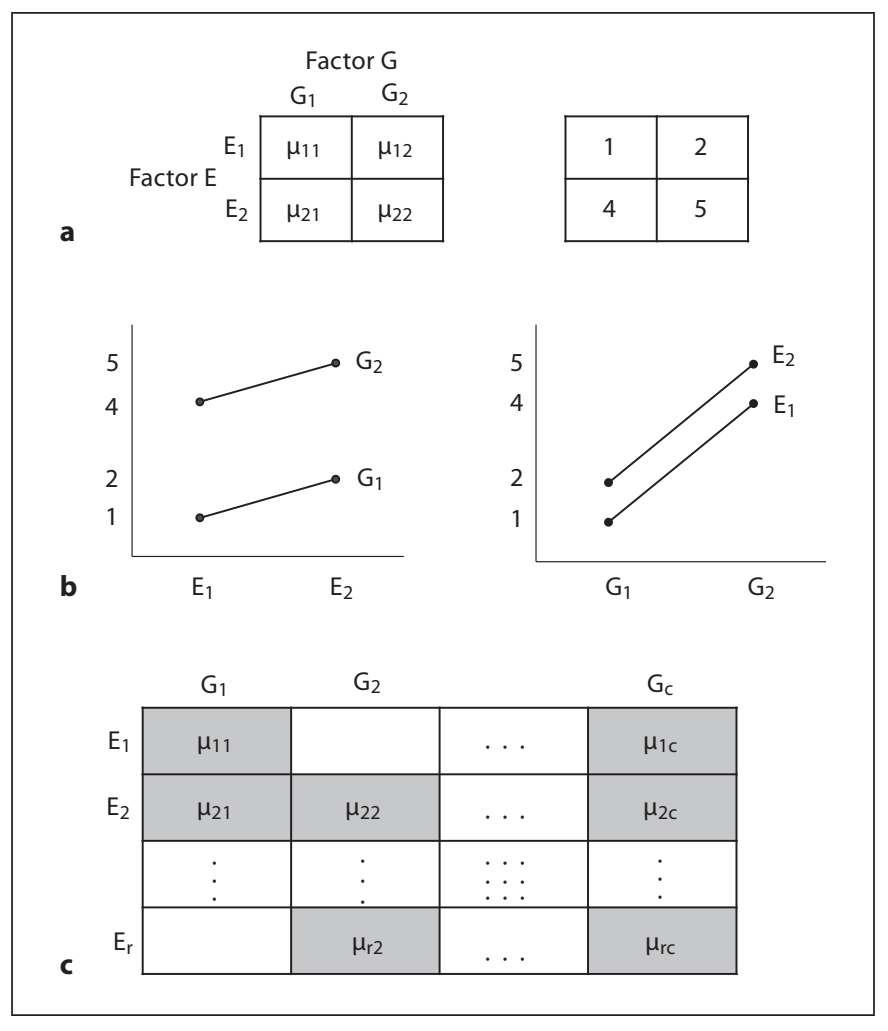

Fig. 2. No interaction. a Left: a $2 \times 2$ table in each cell of which we expect a quantitative outcome; right: mean values that exhibit no interaction. $\mathbf{b}$ Lack of interaction is characterized by two parallel lines. c General $r \times c$ table; there is no interaction if there is 'diagonal equality' for all possible 'rectangle corners' $\left(\mu_{i i}+\mu_{j j}=\right.$ $\mu_{i j}+\mu_{j i}$ - the corners of two such rectangles are highlighted).

cepts - the kind of joint action that is not explained by an additive model, and (3) because genetic epidemiology is quantitative, the terms 'gene-gene interaction' and 'geneenvironment interaction' should only be used for situations that cannot be adequately described by a parsimonious additive statistical model. We now explain the reason for these suggestions.

\section{The Meaning of Statistical Interaction: Back to Basics}

In the following, unless stated otherwise, we are always considering true parameters, not sample estimates. We start by considering the simplest possible case, namely that of two levels of each of two factors, comprising the fourfold table depicted in figure 2a. The factor G may represent genotypes at a locus which we classify into two categories, $G_{1}$ and $G_{2}$. For a diallelic locus with alleles A and $a$, for example, $G_{1}$ could be the two genotypes AA and $\mathrm{Aa}$, and $\mathrm{G}_{2}$ the genotype aa - corresponding to dominance of the allele $A$. Similarly $E_{1}$ and $E_{2}$ could be two categories of genotypes at another locus, or two environments. In each of the four cells we have a distribution of quantitative phenotypes, with means respectively $\mu_{11}$, $\mu_{12}, \mu_{21}$ and $\mu_{22}$. From a statistical perspective, there is no interaction if, and only if, $\mu_{11}+\mu_{22}=\mu_{12}+\mu_{21}$. In other words, the statistical definition of interaction in this simple case is $\mu_{11}+\mu_{22} \neq \mu_{12}+\mu_{21}$. Using, for example, the numbers shown in figure $2 \mathrm{a}$, the lack of interaction is illustrated by the parallel lines in figure $2 \mathrm{~b}$. More generally, if we have a two-way table with $\mathrm{r}$ rows and $\mathrm{c}$ columns, the entry in the $i$-th row and $j$-th column being $\mu_{i j}$, there is no interaction if, and only if, when we consider any four cells in the table that occur at the four corners of a rectangle (fig. $2 \mathrm{c}$ ), we have the equality $\mu_{i i}+$ $\mu_{j j}=\mu_{i j}+\mu_{j i}$. (Here we are implicitly assuming that the phenotypic distributions within each of the four cells differ only in their means; more generally, this equality would be for the four corresponding distributions, not just their means).

Now consider the table of numbers and the corresponding illustration in figure $3 \mathrm{a}$. Here we see clear evidence of interaction. But if we let $x$ denote the values of the means in figure $2 \mathrm{a}$, and $y$ those in figure $3 \mathrm{a}$, we can easily see that $y=2^{x}-1$, which is a monotonic transformation in the range of the four means. Because of this monotonicity, the two lines in figure $3 \mathrm{a}$ do not cross (in contrast to the situation depicted in fig. 4a). Tukey [9] called this type of interaction 'non-additivity' and devised a statistical test to detect it, though this test is sensitive to non-normal residuals [10]. (For a more in-depth discussion of non-additivity, see reference [11]). Thus, Tukey's 1 d.f. test for non-additivity is a test for removable non-additivity, i.e. interaction that can be simply removed by a monotonic transformation [12]. In any situation that such a transformation removes statistical interaction in the sense that we have just defined it, using it will lead to a more parsimonious model under which to conduct a statistical analysis, and hence to more powerful tests for other model parameters [13]. Transforming data this way corresponds to changing the scale of measurement. Note that intersecting lines in an interaction plot, which has been termed 'essential interaction' [14], may nevertheless be largely removable. The cross-over and pure epistatic models (fig. 4b) considered by Chatterjee et al. [13] are examples where there exists a transformation that makes the lines closer to parallel, and hence the in- 


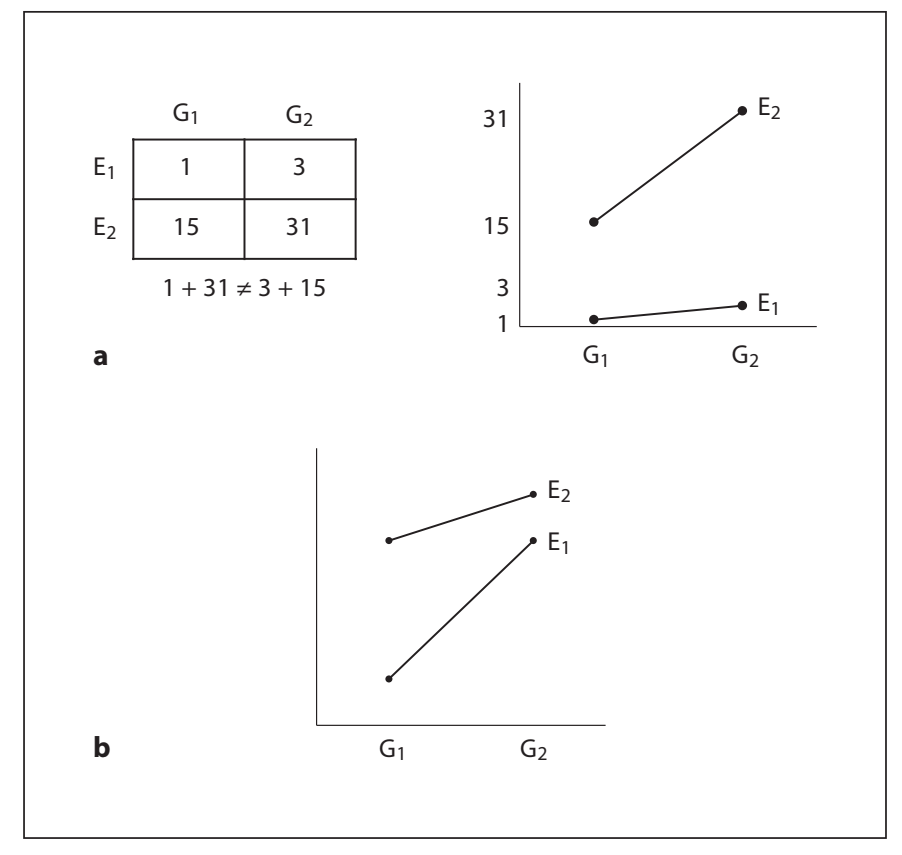

Fig. 3. Removable interaction. Non-parallel lines in a and $\mathbf{b}$ indicate removable interactions. Because the slope of the upper line is greater than the slope of the lower line, $\mathbf{a}$ is often referred to as synergistic interaction. Interactions in $\mathbf{a}$ and $\mathbf{b}$, where the lines have slopes of the same sign and do not cross throughout the ranges considered, can be removed by changing the response scale.

corporation of Tukey's 1 d.f. for non-additivity in the analysis model leads to increased power. Haldane [15] carefully described the different types of gene-environment interaction that are possible.

In a general two-factor model, we can express $\mu_{i j}$ in terms of a linear model such as $\mu_{i j}=\mu+\alpha_{i}+\beta_{j}+\gamma_{i j}$, or redefine $\mu_{i j}=\mu_{i j}-\mu=\alpha_{i}+\beta_{j}+\gamma_{i j}$, where $\mu$ is a general mean, $\alpha_{i}$ and $\beta_{j}$ are main effects, and $\gamma_{i j}$ is the interaction effect. The test for main effects is based on testing the null hypotheses

$$
H_{0}: \alpha_{1}=\alpha_{2}=\ldots=0 \text { and } H_{0}: \beta_{1}=\beta_{2}=\ldots=0 .
$$

The test for interaction effects is based on testing the null hypothesis

$$
H_{0}: \text { all } \gamma_{i j}=\mu_{i j}-\alpha_{i}-\beta_{j}=0 .
$$

As we shall see, whether or not there is interaction in this model can depend critically on the definition of the main effects $\alpha_{i}$ and $\beta_{j}$. There may be empty cells (missing subclasses) in a two-way table, and then the possible tests for main effects and interactions are limited [16]. In samples, empty cells may be caused simply by the limited sample
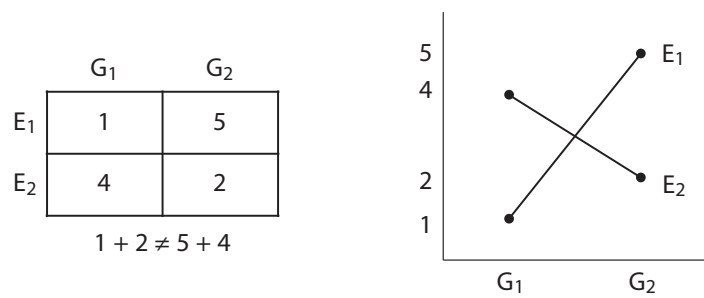

a
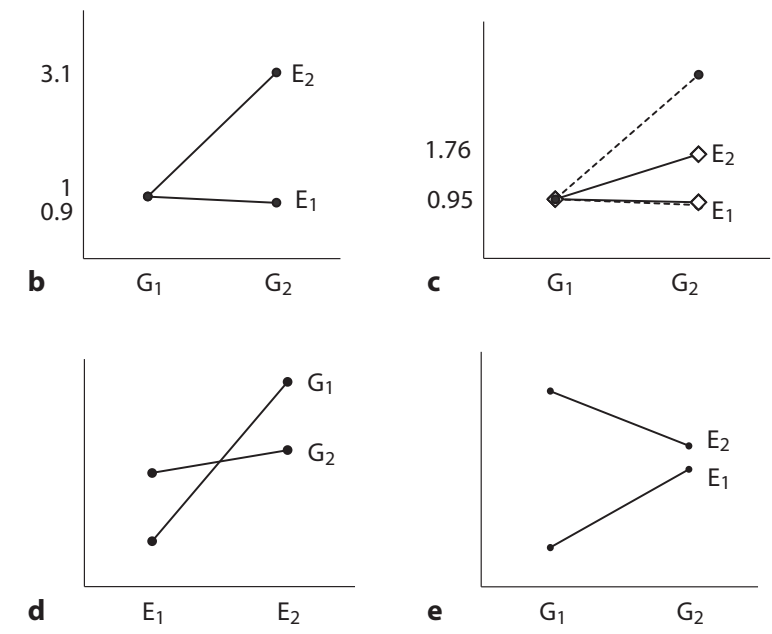

e

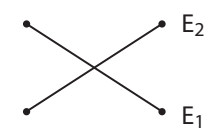

Fig. 4. Non-removable interaction. a Simple example of crossover interaction with crossed lines. b 'Crossover' model used in Chatterjee et al. [13], where the response is the relative risk given the genotypes of two causal loci. Their simulations demonstrated that such a type of interaction can be detected and partially removed by Tukey's 1 d.f. model of interaction. This implies that there exists a monotonic transformation that maximizes the fit of an additive model by making the lines 'more', but not completely, parallel. c illustrates how the original lines (dashed) are changed (solid) when we take a simple square root transformation. This may also apply to other crossover cases where main effects exist, such as the one shown in $\mathbf{d}$; whenever the lines cross, however, the interaction is not completely removable. $\mathbf{e}$ is an alternative plot of the same situation as in $\mathbf{d}$, where the lines do not cross - but note that the slopes have different signs. Whenever the slopes have different signs (d and e), interaction is not completely removable. Interaction is completely 'non-removable' in cases of perfectly antagonistic interaction (zero main effects), as shown in $\mathbf{f}$. 
Fig. 5. Influence of unequal population proportions (weights) on the presence of interaction. We start (top left) with a table of cell means that shows no interaction (as in fig. 2a) and subtract the overall mean, 3 , from each cell mean. If all the cells are weighted equally (unweighted) so that the OR is 1 , then the main row and column effects are as shown and there is no interaction (top right). Similarly, if the weights are proportionate so that the OR is still 1 , we obtain a table (bottom left) in which there is again no interaction. If, however, the weights have an OR of 1.22, we obtain a table (bottom right) in which the interaction effect is non-zero.

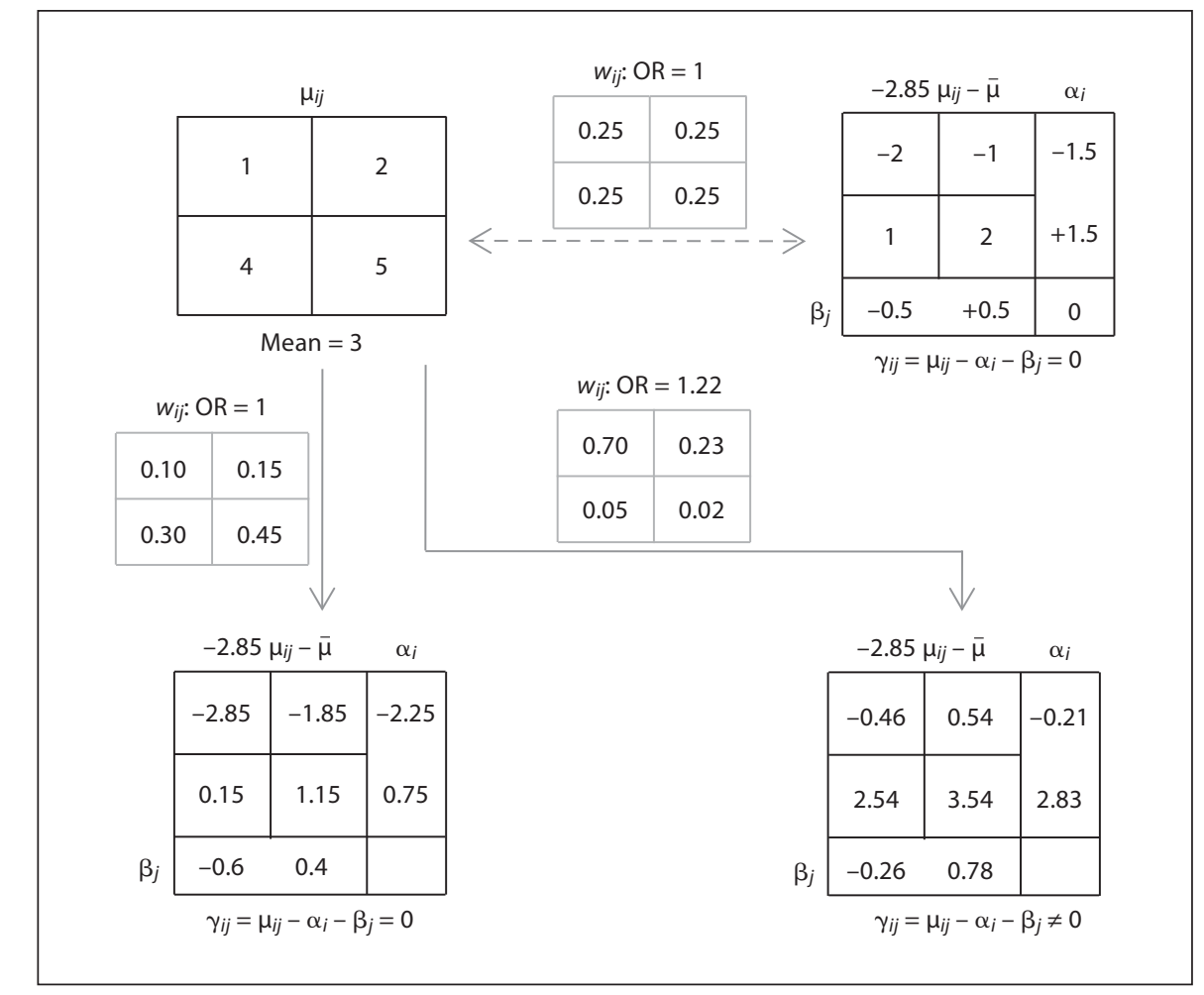

size of the study, because the data obtained are observational rather than from a controlled experiment; but they may also be caused by selection against certain genotypes. Because of this, when analyzing data we need to concern ourselves with another factor that has not been given proper attention - the unequal numbers of observations in cells, known generally in statistics as unbalanced data.

Unbalanced data, like empty cells, can occur either due to chance alone or due to unequal population proportions, which are then true population parameters. When not due to sampling variation, such proportions correspond to allele/genotype frequencies (exposure frequencies) in the case of a gene-gene (gene-environment) study, and their proportions - model parameters - may vary across different populations. When testing for main effects, one has a choice between contrasting unweighted means or weighted means. Unweighted (equally weighted) means are computed as simple averages of the cell means, while weighted means, if we use a common type of analysis, weights the cell means by the cell sizes or proportions. We now focus for the moment on the effect of these two different ways of defining main effects on the estimates of interaction found in samples, using the symbol $w_{i j}$ for the weight given to cell $i j$ (with the sum of the weights over all cells being equal to unity). Mathematically, letting $w_{i j}$ be the proportion of the population in cell $i j$, we define the weighted grand and marginal means respectively as

$$
\bar{\mu}=\sum_{i} \sum_{j} w_{i j} \mu_{i j}, \bar{\mu}_{i .}=\sum_{j} w_{i j} \mu_{i j} / \sum_{j} w_{i j} \text { and } \bar{\mu}_{. j}=\sum_{i} w_{i j} \mu_{i j} / \sum_{i} w_{i j} ;
$$

unweighted means correspond to all the $w_{i j}$ being equal. With these weighted means, the contrasts and tests for main and interaction effects can be set up as in equations (1) and (2). When the main effects are defined using either uniform weights (e.g., $w_{i j}=0.25$ for all four cells of a $2 \times 2$ table) or proportional (meaning that

$w_{i j}=\bar{w}_{i .} \bar{w}_{. j}$, or $w_{i i} w_{j j} / w_{i j} w_{j i}=1$,

corresponding to weights that have an odds ratio (OR) of 1 in a $2 \times 2$ table), the results are the same. But when the weights are unequal and disproportionate (fig. 5), an apparent interaction may result, even if $\mu_{i j}+\mu_{i j}=\mu_{i j}+\mu_{j i}$. Thus, reverting now to population parameters rather than sample estimates, the very meaning of interaction model parameters depends on how the main effects are defined. In the analysis of variance, this is referred to as being due to a non-orthogonal design where the interac- 
tion and main effects are not independent. Thus, the presence of interaction can be induced by giving disproportionate weights to the cell frequencies; and in large samples, as are commonly now used for association studies, except in the case of very rare variants cell frequencies are little affected by sampling variation and thus reflect true population parameters. On the other hand, if there is an interaction in the original data $\left(\mu_{i i}+\mu_{j j} \neq \mu_{i j}+\mu_{j i}\right)$, although assigning different weights may not completely remove the interaction, it will nevertheless have an effect on the magnitude of both main effects and interaction effects.

In human genetics, the phenotype is often dichotomous (e.g., disease or no disease), so that the data are summarized as counts or probabilities (of disease) in each subclass. Let $p_{i j}=$ the probability of disease in cell $i j$ (or penetrance, in the case of two genetic loci). Then everything discussed above applies here also, but with $p_{i j}$ replacing $\mu_{i j}$ in the table. If we transform the penetrance to a $\operatorname{logarithmic~scale,~i.e.,~} \theta_{i j}=\log p_{i j}$, then with equal or proportionate weights, there is no statistical interaction if, and only if, $\theta_{11}+\theta_{22}=\theta_{12}+\theta_{21}$, or $p_{11} p_{22} / p_{12} p_{21}=1$ $(\mathrm{OR}=1)$, corresponding to a multiplicative model on the penetrance scale. Similarly, if we transform using the logit function:

$$
\theta_{i j}=\log \left(\frac{p_{i j}}{1-p_{i j}}\right),
$$

then (again with equal or proportionate weights) there is no interaction when

$$
\left(\frac{p_{11}}{1-p_{11}} \cdot \frac{p_{22}}{1-p_{22}}\right) /\left(\frac{p_{12}}{1-p_{12}} \cdot \frac{p_{21}}{1-p_{21}}\right)=1 \text {. }
$$

This is similar to a multiplicative model for a rare disease; and assuming the two transformations are the same is often what is assumed under 'the rare disease assumption'. But once we take weighted averages, the interpretation of marginal probabilities (i.e. main effects) and interaction changes. In human genetics, gene-gene interaction is in most cases measured on the basis of weighted means. Cheverud and Routman [17] have called the interaction for unweighted means 'physiological epistasis'. They defined all the genetic parameters, including additive and dominance effects, based on unweighted averages of genotypic values, which, unlike the traditional 'main effects' used by statisticians, are independent of genotypic frequencies in the population.

All analyses of statistical interactions are model dependent. An apparent departure from additivity may merely be an artifact of the particular measurement scale, may reflect non-proportional sub-population probabili- ties or may arise from some hidden 'customary' assumptions. A transformation that can remove statistical interaction should always be seriously considered, especially when the most parsimonious model is desirable. There is considerable concern that an improper scale may misrepresent the pattern of response and thus a transformation should not be based merely on 'statistical convenience'. In fact, a rational choice of scale is in most cases difficult or impossible, and there is no definite criterion for a 'true' scale. In a sense, all scales are arbitrary, but some are good if they 'provide the basis of an accurately predictive and usefully descriptive model' [18]. Although the clinical interpretation of results is best made on scales that the clinician is used to, for the purpose of statistical analysis the choice of a scale that minimizes interaction in terms of model parameters can often lead to a more powerful test and more efficient estimates - and then all the results should be transformed back to whatever scale the clinician understands best.

To sum up, interaction as a statistical concept, defined in terms of model parameters, requires the exact definition of the main, or marginal, effects of the factors involved (e.g., whether based on proportional weights or non-proportional weights). Main effects and interactions should always be interpreted together as a system. The term 'pure interaction' and the claim 'interaction can occur without main effects' are somewhat misleading. Conceptually, as we have shown, statistical interactions can only occur after the additivity of main effects has failed to explain the response, which means nothing can be established without first specifying the definition and form of the main effects. When modeling data, interaction terms should not be included in the model without all the corresponding main effect terms, except in very rare instances. It has been demonstrated that omitting a main effect term, even if it is not significant, could lead to severe inferential errors [19]. Finally, the presence of interaction will greatly influence the meaning of the main effects. In the case of perfect antagonism interaction with both marginal effects equal to zero (fig. 4f), the main effects are better interpreted on a conditional basis. An average temperature (of $25^{\circ} \mathrm{C}$ ) makes little sense to a man who has his head in the oven and feet in the freezer.

\section{Gametic Phase Disequilibrium and Interaction}

'Linkage disequilibrium' (LD) is another widely, but often misleadingly, used term in genetics. It is semantically impossible for unlinked loci to be in LD, but un- 
linked loci (even on different chromosomes) can be in gametic phase disequilibrium (GPD). Lewontin, who first introduced the term LD, has written 'I really regret having used the term ... calling it 'gametic phase' rather than 'linkage' disequilibrium would have made that much clearer ...' [R. Lewontin, personal communication]. GPD describes the non-random association of alleles within gametes; and when the alleles involved are in linked loci, we have the special case of LD. The presence of GPD further compounds the complexity of gene-gene interaction, especially for dichotomous traits in a non-experimental organism. For example, if the numbers in each subclass of a two-way table are counts or proportions of diseased people, we cannot distinguish (on the basis of such data alone) whether an extremely high OR is due to a physiological interaction effect or merely caused by disproportionate allele/genotype frequencies, implying GPD. There is thus complete confounding between interaction and GPD. It is well recognized that in the absence of GPD, i.e. when studying independent genotypes, either a case-control or a case-only design can be used to test interactions among loci [20]. But there is very little literature that attempts to investigate how GPD impacts the analysis of interaction, such as when based on logistic regression of case-control data [21]. In genome-wide scans for interacting loci [22], SNP pairs in close physical proximity are often simply filtered out, even though they can easily provide haplotype information. More serious consideration is desirable of the interplay among these excluded SNPs, especially within a gene, because they can be found to be important [23]. In cases where fitness is the trait of interest, gene-gene interaction is believed to be one cause of GPD. However, it has also been shown that interaction between two loci can create different GPD patterns in disease and control populations, and such contrasts can serve as a measure of 'interaction' between two unlinked loci [24], and also between SNPs [25] in GPD.

\section{Concluding Remarks}

Classical genetics, started in 1865 (Mendel) as a statistical science, developed as 'population genetics' with the works of Haldane, Wright and Fisher. During this period, genes were abstractions, defined on the basis of inheritance from one generation to the next. Modern human genetics, though hinted at very early (in 1908 when Garrod proposed the 'one gene, one enzyme' hypothesis) only really became a biochemical science once the structure of DNA became known [26]. At this point, genetics as a field has extended from studying the inheritance of genetic material to encompass, in addition, the physico-chemical process by which that material leads to observable phenotypes. In this sense, in the form of molecular biology, genetics usurped what had until then been classified as, e.g., biochemistry, physiology, embryology, and immunology.

With incomplete physico-chemical knowledge, the gene has been defined as a functional unit, rather than an inherited unit, and until recently the dogma has been asserted that genes code for proteins and that, in humans, these proteins are the only intermediacies between what is inherited (assumed to comprise only the DNA molecules, the rest of the chromosomes being ignored) and the clinical phenotype. Thus, the estimated number of genes in the human genome shrank from over 100,000 to about 21,000 . Now, with more knowledge [4], the gene has been redefined in such a way that humans may well have more than 100,000 genes, whose products interact (physically) in a complicated fashion. But because we still have incomplete knowledge of all the processes involved, human genetics remains to a large extent a statistical science in the study of interactions - and note that, unless physical interactions occur before DNA is transcribed (CTs), the interactions occur between the products of genes, not between the genes themselves. Similarly, gene-environment interaction is practically (at the physical level) a misnomer for interactions between a gene product and some other molecule (perhaps also at origin a gene product) influenced by an environmental factor (chemical, radiation, etc.). This is why we discuss here statistical interaction at length, and what it may or may not mean in terms of molecular interaction, and why it is important to understand in this context the statistical (and epidemiological) concept of confounding as it relates to human studies.

\section{Acknowledgements}

This work was supported in part by the following U.S. Public Health Service grants: Resource grant P41 RR03655 from the National Center for Research Resources; Cancer Center Support grant P30 CAD43703 from the National Cancer Institute; Research grants HL074166 and HL086718 from the National Heart, Lung, Blood Institute; and Research grant HG003054 from the National Human Genome Research Institute. In addition, a grant from the Merck Foundation supported X.W. 


\section{References}

$\checkmark 1$ Cantor RM, Lange K, Sinsheimer JS: Prioritizing GWAS results: a review of statistical methods and recommendations for their application. Am J Hum Genet 2010;86:6-22.

$\checkmark 2$ Musani SK, Shriner D, Liu N, Feng R, Coffey CS, Yi N, Tiwari HK, Allison DB: Detection of gene-gene interactions in genome-wide association studies of human population data. Hum Hered 2007;63:67-84.

$\checkmark 3$ Moore JH: From genotypes to genometypes: putting the genome back in genome-wide association studies. Eur J Hum Genet 2009;17: 1205-1206.

4 Gerstein MB, Bruce C, Rozowsky JS, Zheng D, Du J, Korbel JO, Emanuelsson O, Zhang $\mathrm{ZD}$, Weissman S, Snyder M: What is a gene, post-encode? History and updated definition. Genome Res 2007;17:669-681.

5 Lanctot C, Cheutin T, Cremer M, Cavalli G, Cremer T: Dynamic genome architecture in the nuclear space: Regulation of gene expression in three dimensions. Nat Rev Genet 2007;8:104-115.

6 Bateson W: Mendel's Principles of Heredity. Cambridge, Cambridge University Press, 1909.

7 Fisher R: The correlation between relatives on the supposition of mendelian inheritance. Trans R Soc Edinb 1918;52:399-433.

$\checkmark 8$ Phillips PC: The language of gene interaction. Genetics 1998;149:1167-1171.

9 Tukey JW: One degree of freedom for nonadditivity. Biometrics 1949;5:232-242.
10 Yates F: A Monte-Carlo trial on the behaviour of the non-additivity test with nonnormal data. Biometrika 1972;59:253-261.

$\checkmark 11$ Cox DR, Atkinson AC, Box GEP, Darroch JN, Spjotvoll E, Wahrendorf J: Interaction. Int Stat Rev 1984;52:1-31.

12 Elston RC: On additivity in the analysis of variance. Biometrics 1961;17:209-219.

13 Chatterjee N, Kalaylioglu Z, Moslehi R, Peters U, Wacholder S: Powerful multilocus tests of genetic association in the presence of gene-gene and gene-environment interactions. Am J Hum Genet 2006;79:1002-1016.

14 Wu C, Zhang H, Liu X, DeWan A, Dubrow R, Ying Z, Yang Y, Hoh J: Detecting essential and removable interactions in genome-wide association studies. Start Interface 2009;2: 161-170.

15 Haldane J: The interaction of nature and nurture. Ann Eugen 1946;13:197-205.

16 Elston RC, Bush N: The hypotheses that can be tested when there are interactions in an analysis of variance model. Biometrics 1964 20:681-698.

17 Cheverud JM, Routman EJ: Epistasis and its contribution to genetic variance components. Genetics 1995;139:1455-1461.

18 Eaves LJ, Last K, Martin NG, Jinks JL: A progressive approach to non-additivity and genotype-environmental covariance in the analysis of human differences. Br J Math Stat Psychol 1977;30:1-42.
19 Brambor T, Clark W, Golder M: Understanding interaction models: improving empirical analyses. Polit Anal 2006;14:63-82.

20 Yang Q, Khoury MJ, Sun F, Flanders WD: Case-only design to measure gene-gene interaction. Epidemiology 1999;10:167-170.

21 Cordell HJ: Epistasis: what it means, what it doesn't mean, and statistical methods to detect it in humans. Hum Mol Genet 2002;11: 2463-2468.

22 Emily M, Mailund T, Hein J, Schauser L, Schierup MH: Using biological networks to search for interacting loci in genome-wide association studies. Eur J Hum Genet 2009; 17:1231-1240.

23 Jorgenson E, Witte JS: A gene-centric approach to genome-wide association studies. Nat Rev Genet 2006;7:885-891.

24 Zhao J, Jin L, Xiong M: Test for interaction between two unlinked loci. Am J Hum Genet 2006;79:831-845.

25 Wang T, Zhu X, Elston RC: Improving power in contrasting linkage-disequilibrium patterns between cases and controls. Am J Hum Genet 2007;80:911-920.

26 Watson JD, Crick FHC: Molecular structure of nucleic acids: a structure for deoxyribose nucleic acid. Nature 1953;171:737-738.

27 Phillips PC: Epistasis - the essential role of gene interactions in the structure and evolution of genetic systems. Nat Rev Genet 2008; 9:855-867. 